\title{
Railway car dynamic response to track transition curve and single standard turnout
}

\author{
J. Droździel \& B. Sowiński \\ Warsaw University of Technology, Poland
}

\begin{abstract}
In this paper vehicle dynamic responses to clothoidal, sinusoidal and parabolic types of transition curves are considered. The responses such as vehicle motion and wheel-rail forces are significantly dependent on the type of curve. Also the dynamic interaction between the railway car and a track on a switch and a crossing of a standard turnout is considered. It was made for car-running direction "on" and "from" a switch facing point, and for nominal and geometrically imperfect track. The results imply the conclusion that specific transition curve and turnout parameters create a vital cause of dynamic interaction growth between a vehicle and a track.
\end{abstract}

Keywords: vehicle, track, dynamics, transition curve, standard turnout.

\section{Introduction}

In this paper, the railway car-track system dynamic interaction influenced by track transient excitations is presented. A few transition curves and a single standard turnout are taken into account. Simulation results are obtained and discussed for elastically moveable rails.

Modern railway tracks designed for high speed traffic should meet a number of requirements regarding travel comfort and safety. Designed transition track geometry changes may cause additional dynamic forces which are dependent, among others, on the shape and magnitude of these changes. During track operation the changes are additionally affected by the state of track maintenance. They are also the cause of increased local degradation of track components. For analyzing dynamic processes of this type, an appropriate simulation model was elaborated. 
We have examined some aspects of the effect of track parameters variations on the system reactions. Examinations connected with the estimation of travelling comfort and safety on transition curve and a turnout constituted the basis of these investigations. In the course of applied complex studies, computer simulations were performed to assess the influence of wheelset-track system parameter changes on its dynamic behaviour. Some results of the above mentioned aspects are contained in this approach. The approach extensively examines the idea of the track parameter variations. It refers not only to disperse real values of specific parameters/dimensions regarding theirs nominal values but also to their deviations (e.g. nominal track gauge in relation to its real gauge). The non-symmetry phenomena which can occur in vehicle-track mechanical system are also taken into account (e.g. non-symmetrical rail profiles in the turnout zone).

\section{Rail car-track dynamic system}

The model description is aimed for analysing a rail car running through a transition curve and a standard turnout. The model of vehicle motion along a track consists of two interacting subsystems, namely railway car and track. Track model is subordinated to the area of vehicle-track system analysis. Railway car model (as MBS) consists of inertial solids which are connected by means of massless primary and secondary suspensions. To be exact, it consists of four wheelsets, two bogie frames (including other parts rigidly attached to the wheelset or the frame), and a body. Spring and damping characteristics of the suspensions may be introduced to the system as linear or non-linear. The test includes studying vehicle motion in relation to a reference system that moves along the track axle with the speed of undisturbed vehicle motion. Depending on the geometry of track layout, the reference system may be inertial or non-inertial.

Track model is subordinated to the analysis of dynamics pertaining to lower frequencies. The term of ,lower frequencies" has been adopted from Grassi and Knothe [1] and refers to the range below 20 or $30 \mathrm{~Hz}$. In the low-frequency model, the track is usually a rigid system, with the nominal layout geometry in the form of straight sections, transition curves, circular arcs, crossings and turnouts. Deviations from the nominal track dimensions are described as geometric irregularities (imperfections). Nominal track variable geometry and its deviations are kinematic excitations that influence the wheelsets of the vehicle running along the track. The deviations may also be considered while calculating geometric contact parameters and wheel-rail forces. If the lateral wheel-rail forces are significant, such as on transition curves or turnout, then elastically moveable rails may be introduced. In that case, massless or mass equivalent rail models are adopted. The models co-operate with the car wheelsets. As a result, the equivalent mass of rails under each wheel has 3 degrees of freedom (vertical, lateral, and rotation), Droździel [2].

Assuming that the vehicle moves on a rigid track, the complete model has 27 degrees of freedom (DOF), whereas if the vehicle runs on elastically moveable rails the model has $51 \mathrm{DOF}$. The application of a more complex model ought to 
be conditioned by a preliminary assessment of the significance of track elasticity, because in many cases unmoveable rigid track model is sufficient.

\section{Transition curves}

Let us present three types of transition curves often applied in railway track design. There are clothoidal, parabolic and sinusoidal types. As we know, transition curves are used between straight and curved track or between two adjacent curves to allow gradual change in lateral acceleration. Geometrically they are three dimensional curves with variable curvature and they also introduce variable track cant and, if necessary, gauge widening. The centre line of the transition curve has to fulfil boundary conditions at the connection points of the adjacent track component, Esveld [3]. In this approach we confined numerical results to transition between straight and curved track. Linear variation of the track curvature radius is coupled by differential-integral equation, Sowiński [4]:

$$
\frac{\left(1+\left(y^{\prime}(x)\right)^{2}\right)^{\frac{3}{2}}}{y^{\prime \prime}(x)}=\frac{\int_{0}^{x} \sqrt{1+\left(y^{\prime}(u)\right)^{2}} d u}{l} \rho
$$

where

$x \quad$ - co-ordinate measured along the track centre line $0<x<l$,

$y(x) \quad$ - transition curve in plane (in horizontal projection),

$l \quad$ - transition curve length,

$\rho$ - curvature radius of circular arc.

The result of the solution eqn (1) is a curve called clothoid. The curve gives us linear increase of centrifugal force acting on a vehicle. Also a track cant on a transition curve is introduced as linear function of variable $x: h(x)=h_{o} x / l$, ( $h_{o}$ - track cant on regular arc). But, the above form of cant brings undifferentialability of $h(x)$ on the curve ends. To avoid it, the curve is described using continuity conditions for $h(x)$ and demanding tangent to be zero on both ends of the curve. These conditions can be treated as boundary conditions of finite Bernoulli-Euler beam, Sowiński [4]:

$$
\frac{d^{4} h}{d x^{4}}=0, \quad h(0)=0, \quad h^{\prime}(0)=0, \quad h(l)=h_{0}, \quad h^{\prime}(l)=0
$$

Finally, polynomial function of the 3 rd order is obtained:

$$
h(x)=\frac{h_{0}}{l^{2}} x^{2}\left(3-2 \frac{x}{l}\right)
$$

Now we compare the curve expressed by eqn (2) to the other curves used in track design, Sysak [5]. The first, transition curve of sinusoidal type:

$$
y(x)=\frac{1}{2 \rho}\left[\frac{x^{2}}{2}-\frac{l^{2}}{\pi^{2}}\left(1-\cos \frac{\pi x}{l}\right)\right] ; \quad h(x)=\frac{h_{0}}{2}\left(1-\cos \frac{\pi x}{l}\right)
$$


and the second, a curve of parabolic type of the fourth degree:

$$
y(x)=\frac{1}{2} \frac{x^{4}}{6 l^{2} \rho} ; \quad h(x)= \begin{cases}\frac{2 h_{0}}{l^{2}} x^{2} & 0 \leq x \leq \frac{l}{2} \\ h_{0}-\frac{2 h_{0}}{l^{2}}(l-x)^{2} & \frac{l}{2} \leq x \leq l\end{cases}
$$

It is easy to notice, that transition curves' descriptions in the forms of eqns (2), (3) and (4) formally carry out all the conditions expressed above. Let us consider geometric differences between them. In the beginning, the changes of track curvature and track cant will be compared. Numerical calculations were performed for transition curves which are used between straight and curved track (fig. 1 and 2). The radius of track regular arc was assumed to be $\mathrm{R}=500 \mathrm{~m}$ and track cant $\mathrm{h}_{\mathrm{o}}=0.15 \mathrm{~m}$.

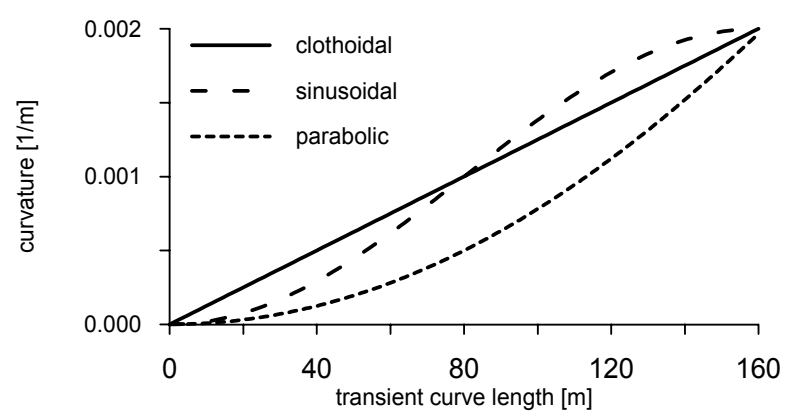

Figure 1: Comparison of track curvature for three transition curves.

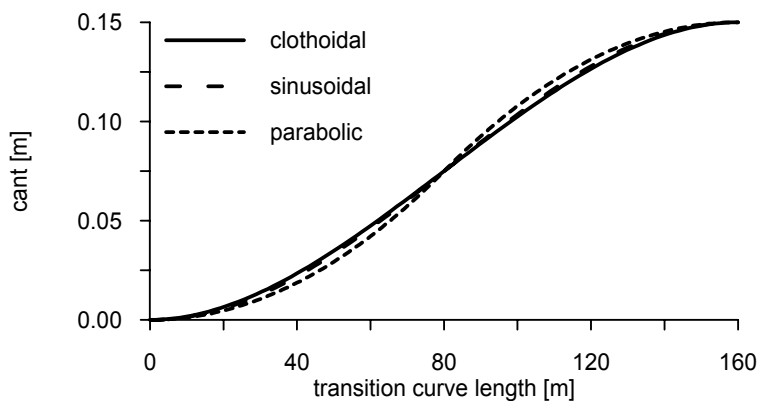

Figure 2: Comparison of cant for three transition curves.

Significant differences are visible first of all for track curvature changes. As a result of it, the differences between vehicle centripetal acceleration for sinusoidal and parabolic curves in relation to clothoidal curve amount to $0.25 \mathrm{~m} / \mathrm{s}^{2}$ at speed of $80 \mathrm{~km} / \mathrm{h}$ (fig. 3). On the basis of rail passenger car simulation [6], these differences really influence the wheelsets dynamic behaviour. 


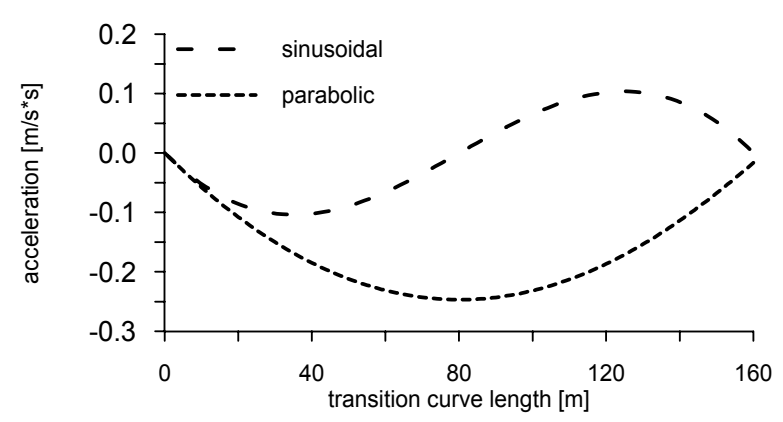

Figure 3: Differences between vehicle centripetal acceleration for sinusoidal and parabolic curves in relation to clothoidal curve.

\section{Single standard turnout}

For transition curves and turnouts, differences between "left" and "right" (outer and inner) rail parameters are observed. One can say that non-symmetry of geometric features occurs. This part deals first of all with modelling them. Vehicle model is to be analysed from the point of view of the influence of that track sections on the forces of interaction between the wheelset and the track and rail car motion.

Standard turnouts may be divided into three typical sections: switch, linking rails, and frog, fig. 4. If the track is analysed as a rigid system, the turnout model is the mathematical description of its geometry. The actual shape and therefore the actual parameters characterising the track in this section are the outcome of its nominal geometry and random deviations that arise during operation. It must be noted, however, that the inertial and spring-damping properties of the rails of a nominal turnout have non-symmetry characteristics as well. For example, the vertical stiffness of the rail on the frog side is greater than that of the other rail.

Starting with a track nominal geometry, the following model assumptions have been accepted, Droździel and Sowiński [7]:

- Unilateral gauge spreading $e(x)$ occurs in the switch and frog areas.

- In the switch area the change of a rail profile is caused by the switch blade adjacent to the stock rail, while in the frog area it is caused by the actual frog point. The vertical cross-section of a track, presented in fig. 5, clearly shows that the profile of a switch blade - 2 adjacent to the stock rail -1 , changes the potential rolling surface of the rail. The combined profile (of switch blade and stock rail) changes as the vehicle's wheelset moves, because the switch blade cross-section enlarges and, at the same time, the stock rail goes away from the nominal track centre line. Finally, switch blade profile reaches the size of a full rail profile, and a track gauge is constant and of standard value. Track parameter changes in the frog area are similar, except that rail discontinuity occurs as well. 


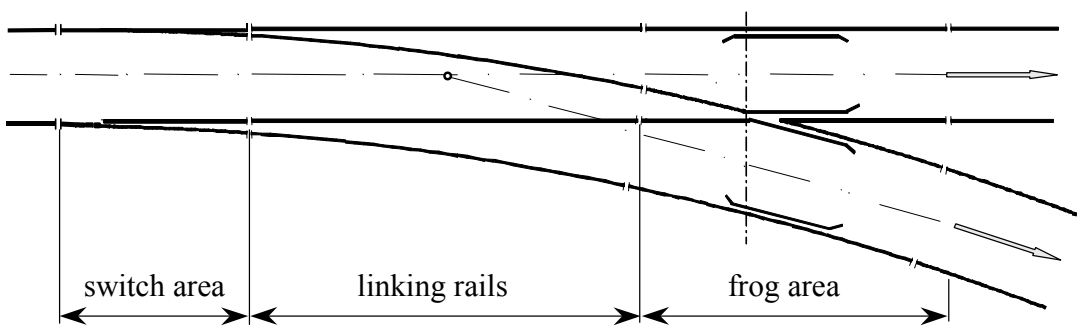

Figure 4: An overview of a single standard right-hand turnout.

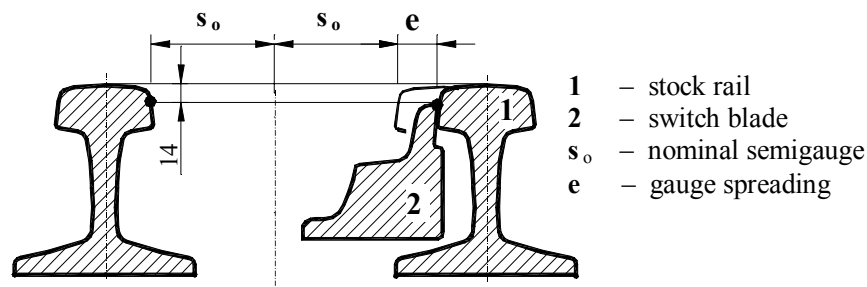

Figure 5: Unilateral gauge spreading $\mathrm{e}(\mathrm{x})$ and non-symmetric rail profiles in the switch area.

- Lateral wheelset motion in the frog area is controlled by check and wing rails. The model presents them as linear-spring non-inertial barriers whose contact with wheel back flanges is the source of extra contact forces.

- When the radius of the diverging track is small, unilateral gauge spreading of a section of the inner linking rail may also be introduced. The model under analysis does not take this factor into account, because the analysis concerns diverging track radii of $R \geq 300 \mathrm{~m}$.

- Apart form the ones mentioned above, there are other characteristics of the object that do not cause non-symmetry in the system and that have a significant effect on vehicle-track interactions. For example, before and after turnouts a change of lateral rail inclination takes place (at turnouts the inclination is equal to zero), and there is no cant on the diverging track.

The formation of track imperfections during operation, e.g. in the form of rail geometric deviations, is different on tracks of constant nominal parameters than in the switch and crossing area. One of the reasons for this is greater dynamic interaction between the vehicle and the track in the turnout area. The phenomenon occurs for a car-running direction "on" and "from" a switch facing point. Therefore, the description of deviations through their reduction to the track centre line equals assuming that, in the model, the deviations of both rails are identical. The above assumption is acceptable only in case of a good quality track and of constant nominal parameters. In other cases, to improve precision, independent introduction of each rail's imperfections is suggested. 


\section{Simulation of rail car motion on transition curve and standard turnout}

In the first part of the paper we present the comparison of leading wheelset lateral displacements and wheel-rail lateral forces for the three transition curves described above. In calculation process measured new wheels of S1002 and new rails of UIC60 profile types were used. They were approximated in preprocessing by cubic spline. All the calculations were performed for vehicle speed of $120 \mathrm{~km} / \mathrm{h}$. Dissimilarities of obtained results are evident, fig. 6 and 7.

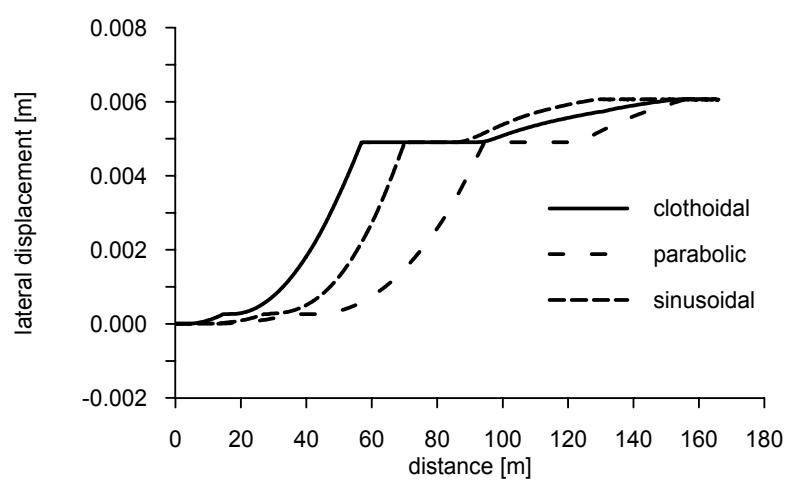

Figure 6: Lateral displacement of leading wheelset centre.

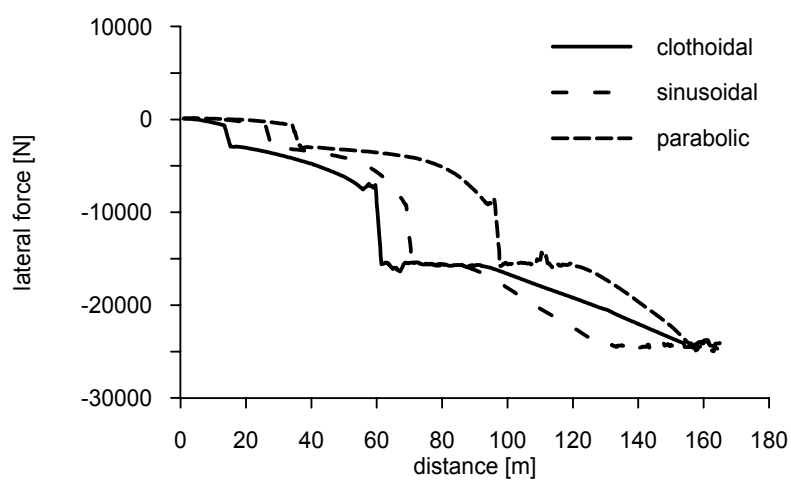

Figure 7: Wheel-rail lateral force acting on outer wheel (leading wheelset).

However, at the end of transition curves the results for displacements and forces becoming close to each other. On the graph of wheelset lateral displacements the effect of wheel profile feature is clearly marked.

Rail car passage throughout the single turnout is a typical example of vehicletrack dynamic system transient process. Numerical simulations were made on diverging track of the single standard turnout (S60-300-1:9 psd, $\mathrm{R}=300 \mathrm{~m})$ at speed of $40 \mathrm{~km} / \mathrm{h}$. Wheel and rail profiles are the same as for simulation runs on 
transition curves. Besides, two cases were considered, that is for car running direction "on" and "from" a switch facing point. In figures 8 and 9 are shown diagrams of simulation results for running on a switch facing point direction.

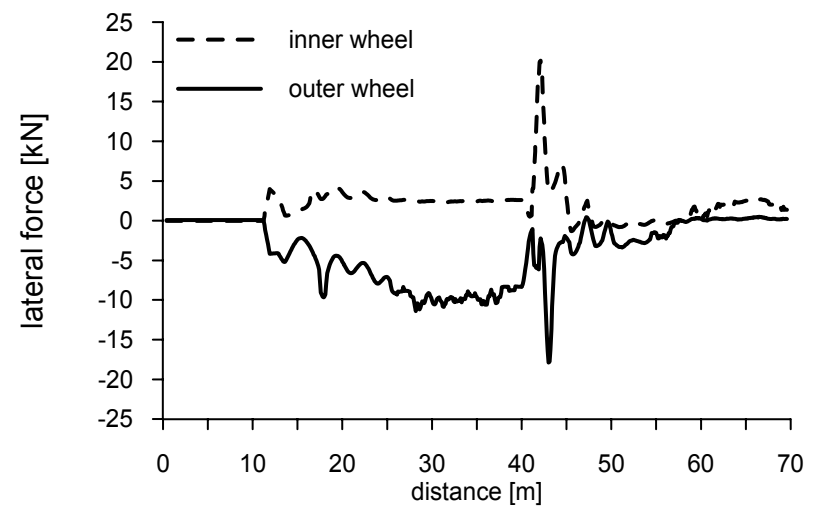

Figure 8: Wheel-rail lateral forces acting on outer and inner wheel. Running on a switch facing point.

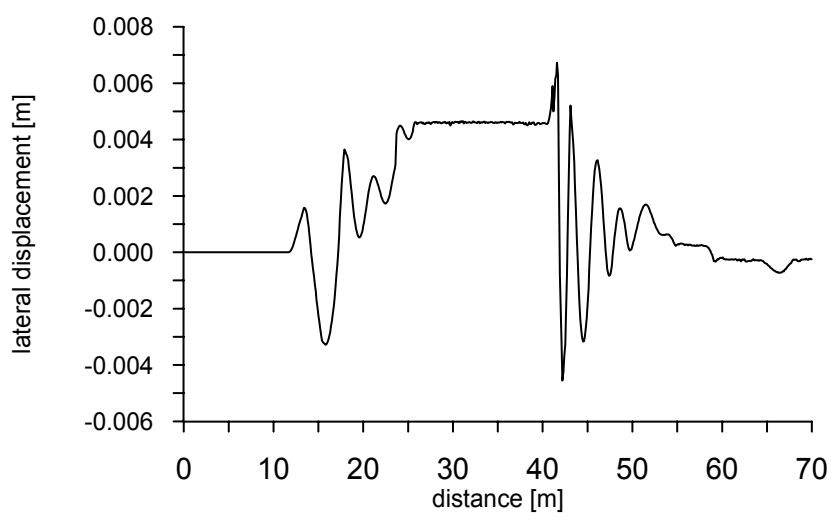

Figure 9: Wheelset centre lateral displacement.

Characteristic properties of the analysed results are the increase of wheel-rail lateral force caused by the change of rail lateral inclination prior to the switch blade and by specific track widening in the switch area. Also, notable increase of a wheelset lateral shift appears in regions of track widening (switch and frog areas). Qualitatively similar diagrams of suitable forces and displacements were obtained for the diverging track with geometric imperfections. It leads to the conclusion that the effect of turnout specific nominal geometry on vehicle dynamics is dominating. Of course, it is valid for good maintained railway track. Appropriate results for vehicle running from a switch facing point are presented in fig. 10 and 11. Additionally, to these results were supplied corresponding diagrams of $2 \mathrm{~mm}$ narrowed track gauge in relation to its nominal dimensions. As 
shown in report [6], vertical and lateral wheel-rail forces, for both runs, on and from switch facing point are not powerfully dependent on track gauge changes in tolerance limit.

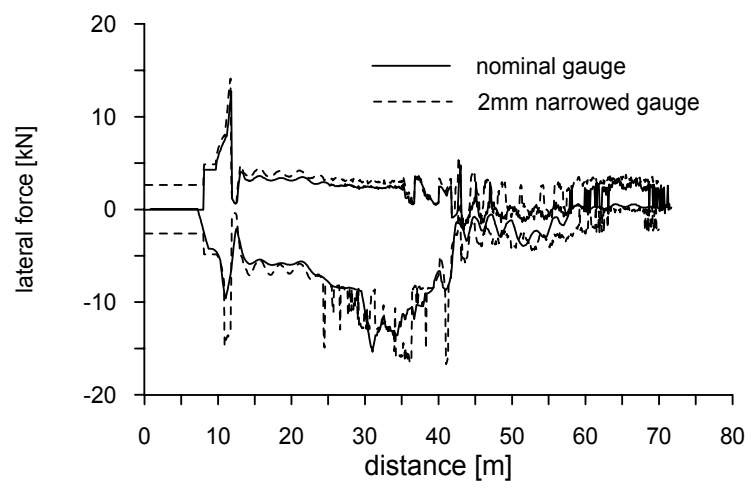

Figure 10: Wheel-rail lateral forces acting on outer and inner wheel. Running from a switch facing point.

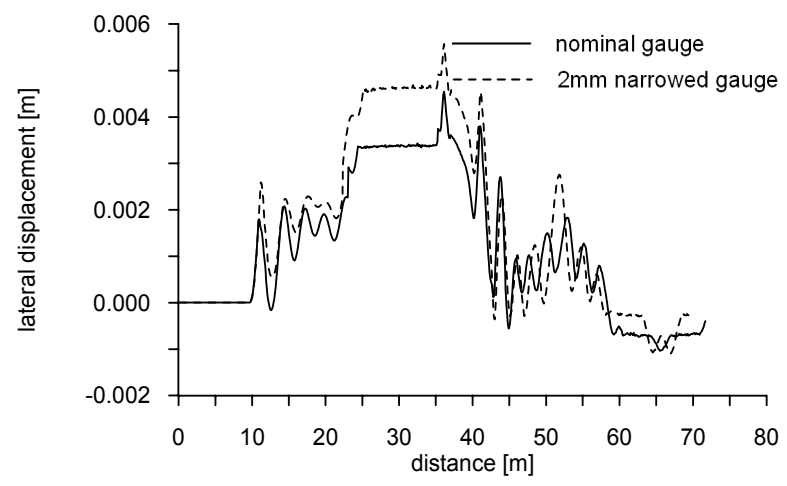

Figure 11: Wheelset centre lateral displacement. Running from a switch facing point.

Generally, they are similar in character to each other. Nevertheless, for runs from a switch direction one can observe stronger wheel-rail dynamic interaction near the switch blade, whereas for runs on a switch, stronger interaction appears in a frog area.

\section{Conclusions}

In the paper we have shown some possibilities of complex simulation model in application to study transient dynamic interaction in the vehicle-track system. It 
has assumed that the interaction is principally affected by track geometric changes. Practical aspect of this study is the opportunity to find answer: what is the weight of that track specific properties on vehicle-track dynamic response and finally on travelling comfort and safety.

\section{Acknowledgement}

The authors wish to acknowledge the partial financial support provided by the State Committee for Scientific Research (Grant KBN No 4 T12C 006 30).

\section{References}

[1] Grassie S.L. Knothe K., Modelling of Railway Track and Vehicle/Track Interaction at High Frequencies. Vehicle System Dynamics, vol 22, pp. 209-262, 1993.

[2] Droździel J., Lateral dynamics of vehicle-track mechanical system with non-symmetric distribution of parameters. Archives of Transport, vol.13, issue 2, Polish Academy of Sciences, Warsaw, pp. 41-56, 2001.

[3] Esveld, K.C., Modern Railway Track, (2 ${ }^{\text {nd }}$ edition). MRT-Productions: The Netherlands, pp. 39-41, 2001.

[4] Sowiński B., Simulation of a Railway Vehicle on Regular Track and Transition Curve. Proc. of $14^{\text {th }}$. Proc. Intern. Conference "Current Problems in Rail Vehicles”, Žilina, Slovakia, pp. 131-138, 1999.

[5] Sysak J. (ed.): Railway Roads. Polish Publishing House (PWN): Warsaw, pp. 219-238, 1982 (in polish).

[6] Droździel J., Sowiński B. et al. Dynamics of transient interaction between running gear of rail vehicle and track. Warsaw University of Technology: Report of Research Project KBN, No 5 T12C 034 22, Warsaw, 2004.

[7] Droździel J., Sowiński B., Computer Simulation of Rail Vehicle-Turnout Dynamic Interaction with Mechanical Imperfections. Proc. $7^{\text {th }}$ International Conference on Computers in Railways-COMPRAIL 2000, eds. J. Allan et al. WIT Press: Southampton, Boston, pp.673-682, 2000. 